\title{
Slowing Down of the Molecular Reorientation of Water in Concentrated Alkaline Solutions
}

\author{
Roberto Cota, Eliane P. van Dam, Sander Woutersen,* and Huib J. Bakker* \\ Cite This: J. Phys. Chem. B 2020, 124, 8309-8316 \\ Read Online
}

ABSTRACT: It is generally accepted that the hydroxide ion $\left(\mathrm{OH}^{-}\right)$is a strong hydrogen bond acceptor and that its anomalously high diffusion constant in water results from a Grotthuss-like structural diffusion mechanism. However, the spatial extent over which $\mathrm{OH}^{-}$ions influence the dynamics of the hydrogen-bond network of water remained largely unclear. Here, we measure the ultrafast dynamics of $\mathrm{OH}$ groups of HDO molecules interacting with the deuterated hydroxide ion $\mathrm{OD}^{-}$. For solutions with $\mathrm{OD}^{-}$ concentrations up to $4 \mathrm{M}$, we find that $\mathrm{HDO}$ molecules that are not directly interacting with the ions have a reorientation time constant of $\sim 2.7 \mathrm{ps}$, similar to that of pure liquid water. When the concentration of $\mathrm{OD}^{-}$ions is increased, the reorientation time constant increases, indicating a strong slowing down of the structural dynamics of the solution.

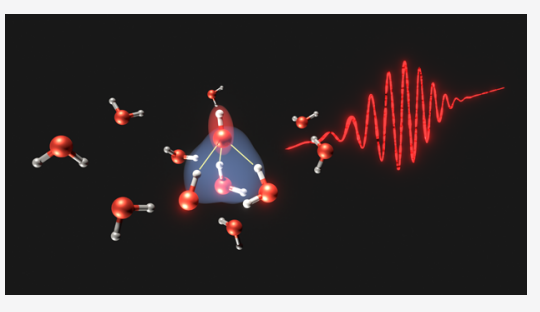

\section{INTRODUCTION}

Proton transfer via hydronium $\left(\mathrm{H}_{3} \mathrm{O}^{+}\right)$and hydroxide ions $\left(\mathrm{OH}^{-}\right)$is of fundamental importance in many chemical and physical processes, ranging from acid-base reactions to proton conducting membranes. ${ }^{1-13}$ Compared to other ions, $\mathrm{H}^{+}$and $\mathrm{OH}^{-}$ions exhibit an anomalously high mobility in aqueous solutions. This high mobility has been explained from a rapid exchange between hydrogen bonds and covalent bonds, leading to charge transfer, the so-called Grotthuss mechanism. ${ }^{14}$ The molecular-scale details of this mechanism remain an active field of experimental and theoretical research. ${ }^{15-27}$

Interestingly, the hydrated proton $\left(\mathrm{H}_{3} \mathrm{O}^{+}\right)$and acid water solutions have been the subject of numerous experimental investigations, whereas experiments on aqueous hydroxide solutions are relatively scarce. ${ }^{28-34}$ Until now, there have only been a few studies on the effect of hydroxide ions on the dynamics of water. ${ }^{29,30,32}$ In these studies, it was found that for hydroxide concentrations up to $3 \mathrm{M}$, the effect on the dynamics of water is negligibly small. ${ }^{29,32}$ In contrast, at a hydroxide concentration of $10 \mathrm{M}$, a very strong effect on water dynamics is observed, ${ }^{30}$ but the time range over which the reorientation could be measured in this experiment was limited $(<2 \mathrm{ps})$. As a result, a clear picture of the effect of high concentrations of hydroxide ions on the dynamics of water is still missing.

One possible reason for the limited number of studies of the effect of $\mathrm{OH}^{-}$ions on the dynamics of water is that the diffusion of aqueous hydroxide ions has long been believed to be the mirror image of that of aqueous protons, showing similar solvation structures as the proton, such as the hydroxide Eigen structure $\mathrm{H}_{7} \mathrm{O}_{4}^{-}\left(\mathrm{HO}^{-} \ldots 3 \mathrm{H}_{2} \mathrm{O}\right)$ and the hydroxide Zundel structure $\mathrm{HO}^{-} \cdots \mathrm{H} \cdots \mathrm{OH}{ }^{35,36}$ However, over the last decades, molecular dynamics simulations indicated that the transfer of aqueous hydroxide ions involves mechanisms that differ from those of the aqueous proton. ${ }^{27,37-42}$ In particular, these simulations have shown that hydroxide ions can form hypercoordinated structures in which the oxygen atom of $\mathrm{OH}^{-}$is coordinated by four water molecules. This structure was found to be quite stable, thereby impeding further transfer, which could be part of the reason why $\mathrm{OH}^{-}$ ions diffuse slower than $\mathrm{H}_{3} \mathrm{O}^{+}$ions. ${ }^{27}$ Until now, conclusive experimental evidence for the presence of this hypercoordinated structure has not been provided.

Ultrafast mid-infrared (IR) spectroscopy is a powerful technique to study molecular structures and inter- and intramolecular interactions with a time resolution down to tens of femtoseconds. These characteristics make ultrafast midIR spectroscopy very well suited to investigate hydrogen-bond dynamics that occur on time scales on the order of (sub)picoseconds. Recently, this technique has been used to study the diffusion and structural rearrangement of $\mathrm{OH}^{-}$ions in $\mathrm{NaOH}$ (and $\mathrm{NaOD}$ ) solutions..$^{31,33,34,43}$ By probing the dynamics of $\mathrm{OH}$ groups that directly interact with the $\mathrm{OH}^{-}$ ions, it was found that the transfer of the hydroxide charge is relatively slow, with a lower bound of $3 \mathrm{ps,} \mathrm{compared} \mathrm{to} \mathrm{the}$ time scale $<2$ ps that has been observed for $\mathrm{H}_{3} \mathrm{O}^{+}$ions. ${ }^{20,21}$ While these experimental studies provided information on the rate and mechanism of the $\mathrm{OH}^{-}$ion transfer in aqueous media, the influence of the $\mathrm{OH}^{-}$ions on the dynamics of the

Received: April 23, 2020

Revised: August 25, 2020

Published: August 25, 2020 
hydrogen-bond network of water remains practically unexplored. $^{30,44}$

The reorientation dynamics of water molecules in aqueous solution can be well studied with polarization-resolved femtosecond vibrational spectroscopy. ${ }^{45-49}$ In this technique, the reorientation dynamics of a vibrationally excited $\mathrm{OH}$ or $\mathrm{OD}$ group of a $\mathrm{HDO}$ molecule in isotopic dilution (OD in $\mathrm{H}_{2} \mathrm{O}$ or $\mathrm{OH}$ in $\mathrm{D}_{2} \mathrm{O}$ ) is measured. Irrespective of whether the $\mathrm{OH}$ or $\mathrm{OD}$ group is probed, the measured reorientation dynamics are representative for the dynamics of all water molecules present in solution. For the study of the dynamics of water in hydroxide solutions, the two isotopic alternatives have their advantages and disadvantages. The OD vibration of HDO dissolved in $\mathrm{H}_{2} \mathrm{O}$ has the advantage that its excitation lifetime of $T_{1, \mathrm{OD}}=1.8 \mathrm{ps}$ provides a quite large time window (typical 5-6 times $T_{1}$ ) to study molecular dynamics and bonding interactions. ${ }^{50}$ However, the addition of $\mathrm{OH}^{-}$ions gives rise to background absorption that could mask the OD stretch band ${ }^{30,51}$ and may induce more complex relaxation mechanisms. ${ }^{52}$ In this respect, the $\mathrm{OH}$ vibration in $\mathrm{D}_{2} \mathrm{O}$ is a better choice because it is spectrally well-isolated from the continuum that results from the interaction of $\mathrm{D}_{2} \mathrm{O}$ with $\mathrm{OD}^{-}$, as shown in Figure 1. A disadvantage of probing the $\mathrm{OH}$ vibration is that

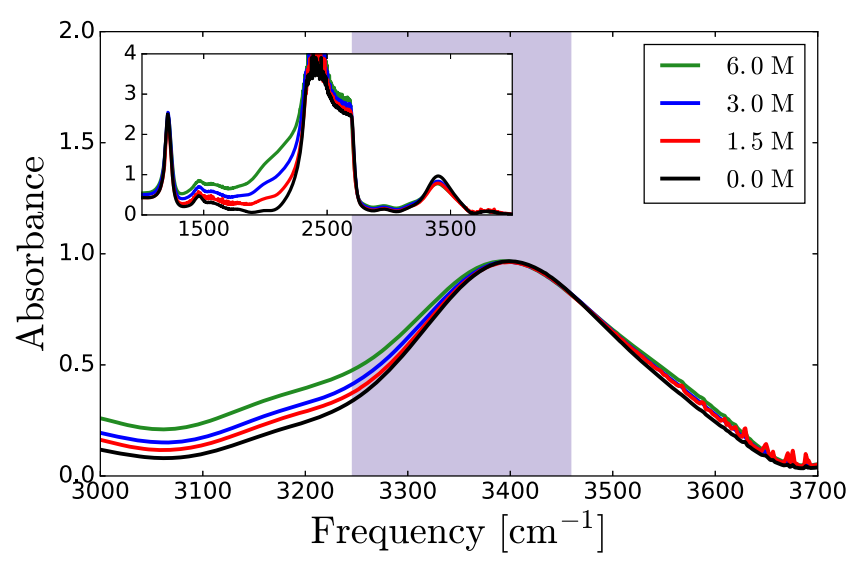

Figure 1. Linear IR spectra of $8 \%$ HDO isotopically diluted in $\mathrm{NaOD} / \mathrm{D}_{2} \mathrm{O}$ solutions at four different concentrations. The spectra are normalized to the maximum of the $\mathrm{OH}$ stretch peak at $3400 \mathrm{~cm}^{-1}$ in neat $\mathrm{D}_{2} \mathrm{O}$. The low frequency shoulder is assigned to $\mathrm{OH}$ groups of $\mathrm{HDO}$ molecules that form strong hydrogen bonds with $\mathrm{OD}^{-}$ions, while the high frequency shoulder $\mathrm{OH}^{-}$ions are weak hydrogen-bond donors. The inset shows the deuteroxide continuum far from the $\mathrm{OH}$ spectral region. The purple-shaded bar indicates the region where the transient absorption spectra were measured.

the study of the reorientation dynamics is limited to a short time interval because of the relatively fast relaxation of the excited $\mathrm{OH}$ stretch vibration, $T_{1, \mathrm{OH}}=0.7 \mathrm{ps} .{ }^{53}$ Here, we present a study of the reorientation dynamics of water molecules in a series of solutions of sodium deuteroxide in $\mathrm{D}_{2} \mathrm{O}$ by measuring the dynamics of the $\mathrm{OH}$ stretch vibration of HDO molecules.

\section{EXPERIMENTAL SECTION}

We performed polarization-resolved femtosecond mid-IR transient absorption measurements using light pulses centered at $3390 \mathrm{~cm}^{-1}$ at $1 \mathrm{kHz}$ repetition rate. The mid-IR pulses are generated via optical parametric down amplification using a KTA crystal (Orpheus-ONE-HP, Light Conversion). This process results in a tunable signal $(1350-2060 \mathrm{~nm})$ and a tunable idler $(2060-4500 \mathrm{~nm})$. At $3390 \mathrm{~cm}^{-1}$, the pulses have an energy of $25 \mu \mathrm{J}$, a bandwidth of $90 \mathrm{~cm}^{-1}$, and a pulse duration of $280 \mathrm{fs}$. A small portion of the generated IR beam is split with a $\mathrm{ZnSe}$ beamsplitter to create the probe. Using a zero-order $\lambda / 2$ waveplate, the polarization of the pump beam is set at $45^{\circ}$ with respect to the probe beam. After the sample, the parallel or the perpendicular component of the probe is chosen using a wire grid polarizer.

The pump pulse promotes a few percent of $\mathrm{OH}$ stretch vibrations from the ground state to the first excited vibrational state, $|0\rangle \rightarrow|1\rangle$. This excitation leads to stimulated emission and a reduced absorption of the probe pulse, thus leading to enhanced transmission of the probe (bleach). We thus detect a transient absorption signal $\Delta \alpha\left(=\ln \left[T_{0} / T\right]\right)<0$, with $T / T_{0}$ the pumped-unpumped transmission ratio of the sample. The probe pulse can also further excite the excited $\mathrm{OH}$ vibration to the second excited state $|2\rangle$, thus giving rise to an induced absorption signal (positive absorption change) at lower frequencies (defined by the anharmonicity of the $\mathrm{OH}$ stretch vibration), that is, $\Delta \alpha\left(=\ln \left[T_{0} / T\right]\right)>0$.

The pump will preferentially excite $\mathrm{OH}$ stretch vibrations that have their transition dipole moments oriented parallel with the pump polarization. Hence, the excitation results in an anisotropic distribution of excited oscillators. This anisotropy can be probed with probe pulses that have their polarization parallel and perpendicular to that of the pump pulse. Immediately after the excitation, the anisotropy will be high, but over time, the excited HDO molecules will show orientational diffusion making the excitation distribution increasingly isotropic. Consequently, the change of the anisotropy as a function of time provides information on the molecular reorientation. The anisotropy is defined as

$$
R=\frac{\Delta \alpha_{\|}-\Delta \alpha_{\perp}}{\Delta \alpha_{\|}+2 \Delta \alpha_{\perp}}
$$

where the denominator is the so-called isotropic signal that depends only on vibrational energy relaxation and spectral diffusion

$$
\Delta \alpha_{\text {iso }}=\frac{\Delta \alpha_{\|}+2 \Delta \alpha_{\perp}}{3}
$$

\section{RESULTS}

Linear IR Spectra. We prepared deuteroxide solutions by diluting a commercially available solution of $40 \mathrm{wt} \% \mathrm{NaOD}$ (Sigma-Aldrich products) in $\mathrm{D}_{2} \mathrm{O}(99.96 \%)$. We add $\mathrm{HDO}$ keeping the $\mathrm{OH} / \mathrm{OD}$ ratio constant at $4 \%$ for all studied solutions. The sample cell consists of two parallel $\mathrm{CaF}_{2}$ windows separated by a Teflon spacer of $50 \mu \mathrm{m}$.

Figure 1 displays linear absorption spectra of the HOD/ $\mathrm{NaOD} / \mathrm{D}_{2} \mathrm{O}$ solutions. The main band around $3400 \mathrm{~cm}^{-1}$ is assigned to the $\mathrm{OH}$ stretch vibration. With increasing $\mathrm{OD}^{-}$ concentration, a shoulder at lower frequencies rises that we assign to $\mathrm{OH}$ groups of $\mathrm{HDO}$ molecules forming strong hydrogen bonds to $\mathrm{OD}^{-}$ions. There is a weak shoulder in the blue wing at $3600 \mathrm{~cm}^{-1}$ that also rises with increasing $\mathrm{OD}^{-}$ concentration. This shoulder is attributed to the stretch vibration of $\mathrm{OH}^{-}$ions that are weak hydrogen-bond donors. The inset in Figure 1 shows that the vibrations around 3400 $\mathrm{cm}^{-1}$ are spectrally well isolated from the continuum arising from the interactions of the abundant $\mathrm{D}_{2} \mathrm{O}$ molecules with $\mathrm{OD}^{-}$ions. 
Vibrational Relaxation Dynamics. Figure 2 shows isotropic transient absorption spectra in the spectral region
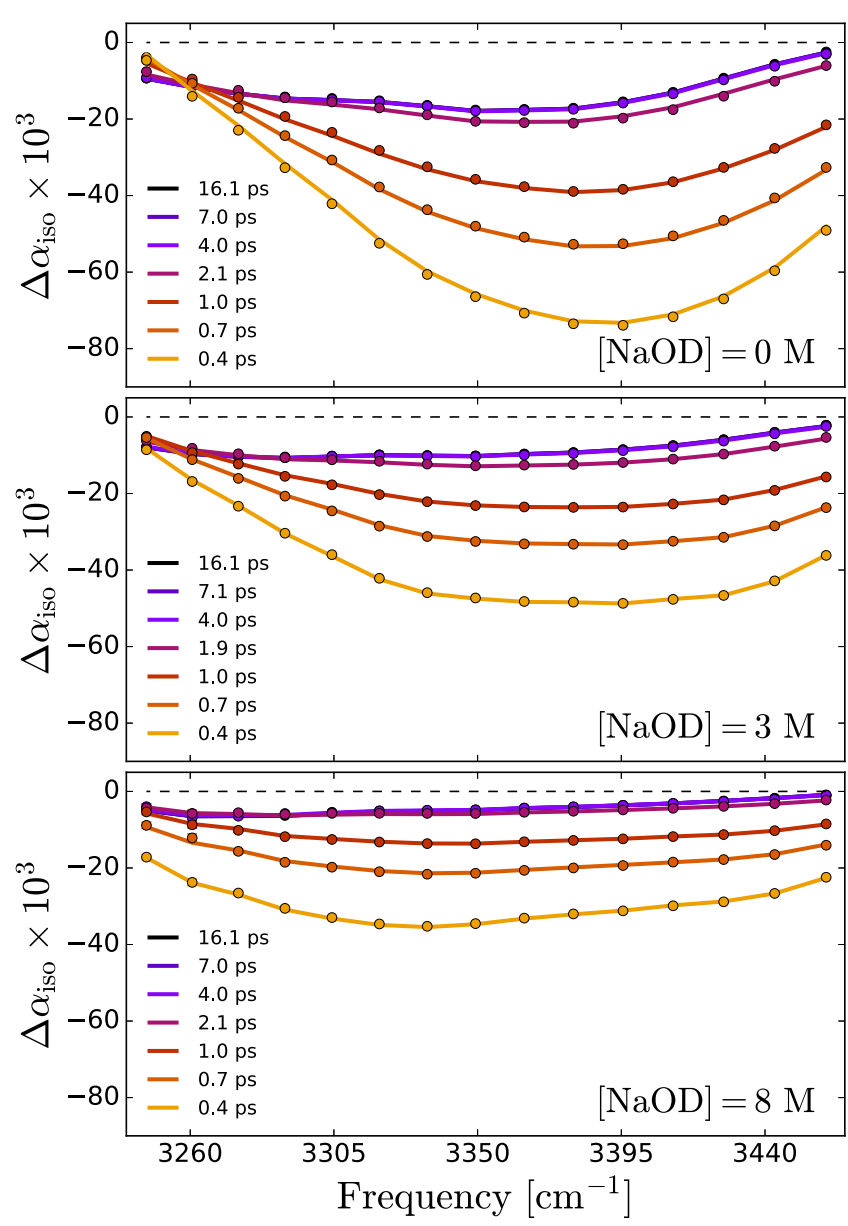

Figure 2. Transient spectra of aqueous solutions of $\mathrm{NaOD}$ in $\mathrm{D}_{2} \mathrm{O}$ at 0,3 , and $8 \mathrm{M}$ concentrations. All samples contain $8 \% \mathrm{HDO}$. The dots indicate the experimental data, while solid lines are fits to the relaxation model described in the main text.

of the $\mathrm{OH}$ stretch vibrations measured at different delays for three different $\mathrm{OD}^{-}$concentrations. We only show spectra from delays of 0.4 ps onwards as the spectra at earlier times show effects of spectral diffusion ${ }^{54}$ and coherent coupling of the pump and probe pulses. The transient spectra are dominated by the bleaching of the fundamental $|0\rangle \rightarrow|1\rangle$ transition. In the low-frequency region, we observe the effect of induced absorption of the $|1\rangle \rightarrow|2\rangle$ transition. ${ }^{50}$

At delay times $>5 \mathrm{ps}$, the vibrational excitation has relaxed, and the energy has thermalized over the focus of the sample leading to a local temperature increase (on the order of a few $\mathrm{K}){ }^{55}$ This heating leads to a decrease and blue-shift of the absorption spectrum of $\mathrm{OH}$ vibration, which corresponds to a long-living bleaching effect in the red wing of the transient absorption spectra of Figure 2.

In modeling the vibrational dynamics of the excited $\mathrm{OH}$ vibration of $\mathrm{HDO}$ molecules dissolved in $\mathrm{NaOD} / \mathrm{D}_{2} \mathrm{O}$ solutions, we take into account the fact that the transient spectra result from the excitation of two different species: the $\mathrm{OH}$ vibration of $\mathrm{HDO}$ molecules that donate a hydrogen bond to the oxygen of a surrounding water molecule, with an absorption band centered at $3420 \mathrm{~cm}^{-1}$, and the $\mathrm{OH}$ vibration of $\mathrm{HDO}$ molecules $\mathrm{H}$-bonded to $\mathrm{OD}^{-}$, with an absorption band centered at $3340 \mathrm{~cm}^{-1}$. These bands refer thus to $\mathrm{OH}$ oscillators in the first solvation shells of $\mathrm{OD}^{-}$ions and to $\mathrm{OH}$ oscillators outside the first solvation shell, which we will refer to in the following as bulk $\mathrm{OH}$ oscillators, although these latter oscillators cannot be rigorously denoted as bulk when the concentration of $\mathrm{OD}^{-}$is very high. Previous studies showed that the heating effect is often delayed in comparison to the relaxation of the $\mathrm{OH}$ stretch vibration. ${ }^{53,55}$ This delay has been explained from the relatively slow adaptation of the hydrogenbond network to the local energy dissipation resulting from the vibrational relaxation. The isotropic signal shows a negligible further evolution after 5 ps, as shown in the inset of Figure 3, which implies that the system has fully relaxed.

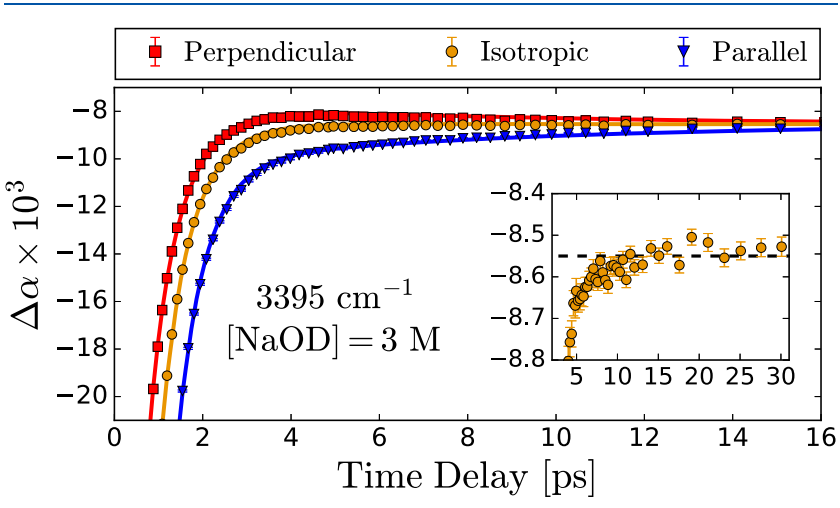

Figure 3. Parallel (blue), isotropic (yellow), and perpendicular (red) absorption change $\Delta \alpha$ as a function of delay time following the excitation of the $\mathrm{OH}$ vibration of $\mathrm{HDO}$ in a $3 \mathrm{M} \mathrm{NaOD} / \mathrm{D}_{2} \mathrm{O}$ solution. The inset zooms in the isotropic signal at long delay times. The solid lines are fits to the models presented in the text.

Based on the above considerations, we arrive at the kinetic model shown in Figure 4. This model comprises two initially

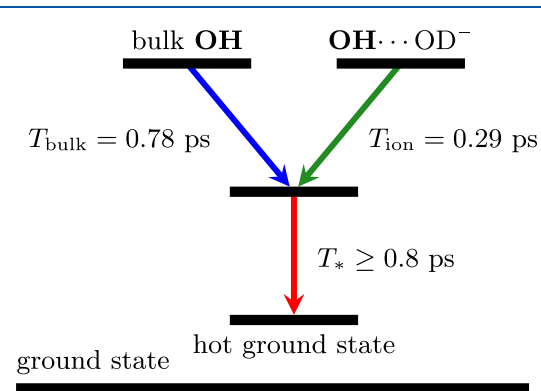

Figure 4. Schematic energy level diagram of the model that describes the vibrational dynamics of hydroxyl groups around $3400 \mathrm{~cm}^{-1}$. Solid arrows indicate the channels for vibrational relaxation. The intermediate step represents the delayed adaptation of the hydrogen-bond network to the resulting vibrations from the fast relaxation process. The details of the model are described in the text.

excited $\mathrm{OH}$ species that relax via an intermediate state to a hot ground state. The transitions between the different components are described with the following set of differential equations

$$
\frac{\mathrm{d}}{\mathrm{d} t} N_{i}(t)=K_{i j} N_{j}(t)
$$

where $N_{i}(t)$ is the population dynamics, $K_{j i}$ is the rate matrix that contains the transition rates according to the kinetic model, and the labels $i$ and $j$ refer to the different states 
considered in the kinetic relaxation model. The rate matrix corresponding to the relaxation mechanism of Figure 4 is

$$
K_{i j}=\left[\begin{array}{llll}
-k_{\text {bulk }} & 0 & 0 & 0 \\
0 & -k_{\text {ion }} & 0 & 0 \\
+k_{\text {bulk }} & +k_{\text {ion }} & -k_{*} & 0 \\
0 & 0 & +k_{*} & 0
\end{array}\right]
$$

where $k_{\text {bulk }}$ and $k_{\text {ion }}$ are the decay rates of bulk and ionassociated $\mathrm{OH}$ excitations, and $k_{*}$ is the decay rate of the intermediate state to the hot ground state. Each decay rate corresponds to a time constant, $T_{i}=1 / k_{i}$.

Each vibrational level has an associated transient absorption spectrum, $\sigma_{i}(\omega)$, and the total isotropic transient signal is given by

$$
\Delta \alpha_{\text {iso }}(t, \omega)=\sum_{i=1}^{n} N_{i}(t) \sigma_{i}(\omega)
$$

where $n$ is the number of levels. Notice that the intermediate state has no associated transient absorption difference spectrum: this state only serves to account for the delayed rise of the signal of the hot ground state.

By combining eqs 3-5, the energy relaxation time constants and the spectral components are obtained via a least-squares minimization process (see Supporting Information). In order to reduce the number of fitting parameters, the spectrum of the thermal ground state is extracted from the spectra at late delay times. Hence, the only fitted parameters are the spectral traces, $\sigma_{\text {bulk }}$ and $\sigma_{\text {ion }}$ at each concentration. The decay time constants, $T_{\text {bulk }}$ and $T_{\text {ion }}$ are treated as global fitting parameters, meaning that they are assumed to be the same at all concentrations. Figure 5 shows the results of the spectral decomposition of the

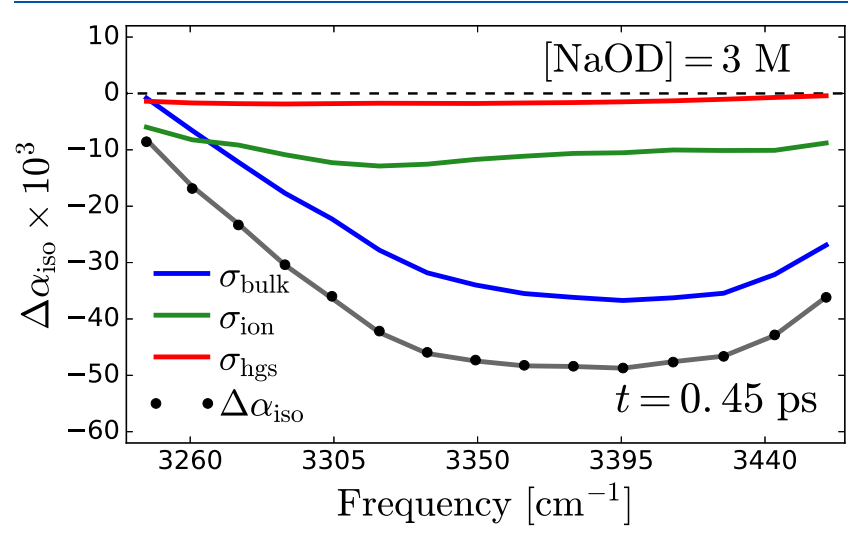

Figure 5. Spectral decomposition of the transient absorption spectrum measured at 0.45 ps delay time for a solution of HDO and $3 \mathrm{M} \mathrm{NaOD}$ in $\mathrm{D}_{2} \mathrm{O}$ solution. The black circles represent the experimental data, and the gray curve results from a least-square optimization of the model given by eqs $3-5$ to the experimental data.

transient absorption spectrum measured at 0.45 ps delay time for a solution of $\mathrm{HDO}$ and $3 \mathrm{M} \mathrm{NaOD}$ in $\mathrm{D}_{2} \mathrm{O}$ (the fit and spectral decomposition at different concentrations are provided in the Supporting Information). The shapes of $\sigma_{\text {bulk }}$ and $\sigma_{\text {ion }}$ show very little change when the $\mathrm{OH}^{-}$concentration is varied, but their amplitudes strongly depend on the concentration. The fitted decay rates correspond to time constants of $0.78 \pm 0.05$ and $0.29 \pm 0.05$ ps for the bulk and ion-associated $\mathrm{OH}$ vibrations, respectively. The time constant of the intermediate state, $T_{*}$, expressing the delayed rise of the thermal effect, has a value of $\sim 0.8$ ps at the lowest $\mathrm{OD}^{-}$ concentration. This time constant slightly increases with an increasing $\mathrm{OD}^{-}$concentration (see Supporting Information), probably as a result of the hydrogen-bond network becoming more rigid at high concentrations.

In the above description, we did not consider the possibility of energy exchange between the two initially excited $\mathrm{OH}$ species. However, recent experiments have shown that the bulk and ion-associated bands exchange energy with a time constant of $\sim 9$ ps. ${ }^{56}$ This energy exchange is much slower than the vibrational relaxation of the bulk and ion-associated $\mathrm{OH}$ vibrations, which implies that the energy exchange will have a negligible effect on the overall relaxation dynamics.

Reorientation Dynamics. Figure 3 shows the parallel and perpendicular absorption change for a $3 \mathrm{M} \mathrm{NaOH} / \mathrm{D}_{2} \mathrm{O}$ solution. We observe that the parallel and perpendicular signals differ at delay times at which the isotropic signal has fully relaxed, that is, it has become fully thermal. This residual anisotropy indicates that the final relaxed state has retained some memory of the orientation of the originally excited state. This result shows that the vibrational relaxation is fast compared to the characteristic reorientation time of the excited molecules and the global energy redistribution time. In all investigated samples, the vibrational energy relaxation time constants are significantly shorter than the characteristic reorientation time of water of $\sim 2.6$ ps. With increasing $\mathrm{OD}^{-}$ concentration, the fast ion-associated relaxation pathway becomes more dominant, which increases the residual anisotropy of the hot ground state.

We use the information obtained from the modeling of the isotropic data to model the anisotropy data. Using eqs 1,2 , and 5 , the parallel and perpendicular transient absorption can be written in terms of the characteristic reorientation and relaxation mechanisms for each $i$-component as

$$
\begin{aligned}
& \Delta \alpha_{\|}(t, \omega)=\sum_{i=1}^{m}\left[1+2 R_{i}(t)\right] N_{i}(t) \sigma_{i}(\omega) \\
& \Delta \alpha_{\perp}(t, \omega)=\sum_{i=1}^{m}\left[1-R_{i}(t)\right] N_{i}(t) \sigma_{i}(\omega)
\end{aligned}
$$

We can now associate an anisotropy dynamics function $R_{i}(t)$ with each level. We assume that for all levels the anisotropy dynamics follows a monoexponential decay, as

$$
R_{i}(t)=A_{i} \mathrm{e}^{-t / \tau_{\mathrm{or}, i}}
$$

The decay time constant, $\tau_{\mathrm{or}, i}$ of each anisotropy component $R_{i}$, is obtained from a global least-squared analysis of the transient absorption data measured in parallel and perpendicular polarization configurations (see Supporting Information). We use several physical considerations to limit the number of free parameters. The first consideration is that the initial anisotropy of all levels cannot exceed a maximum theoretical value of $R(0)=2 / 5$. We also take the initial anisotropy $A_{\text {bulk }}$ as a concentration-independent global parameter. The solid curves in Figures 3 and 6 represent the resulting fits, which describe the data very well. We find that the contribution to the total anisotropy of ion-associated $\mathrm{OH}$ groups is negligible from 0.4 ps onwards because of its extremely short vibrational relaxation time. The reorientation time constant $\tau_{\text {or,hgs }}$ associated with the hot ground state is significantly larger 


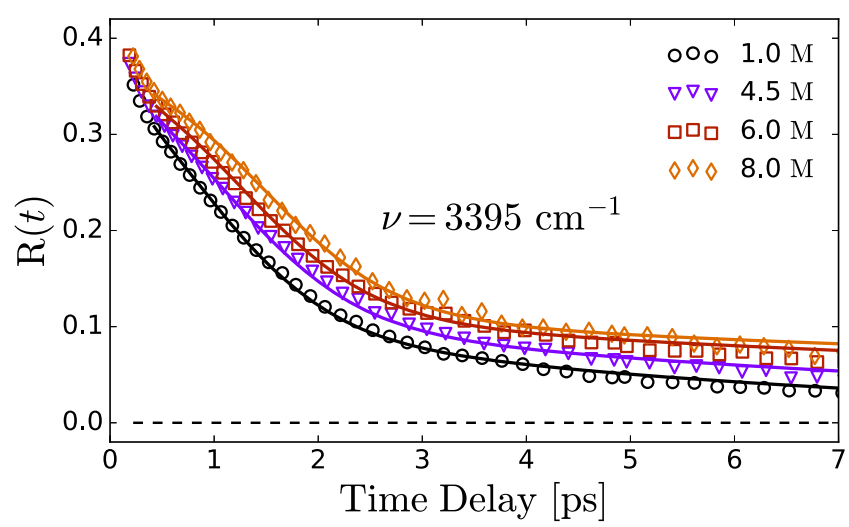

Figure 6. Anisotropy as a function of delay time for $\mathrm{NaOH} / \mathrm{D}_{2} \mathrm{O}$ solutions. The solid lines are fits to the experimental data via the model described in the main text.

than $\tau_{\text {or,bulk }}$ over the whole concentration range. It increases from $6.0 \pm 0.4 \mathrm{ps}$ at low concentrations to $20 \pm 3$ ps at a solution of $8 \mathrm{M}$ (see Supporting Information). This implies that $\mathrm{OD}^{-}$ions stiffen the hydrogen bond network.

Figure 7 shows that the reorientation time constant of bulklike $\mathrm{OH}$ groups increases with increasing $\mathrm{OD}^{-}$concentration,

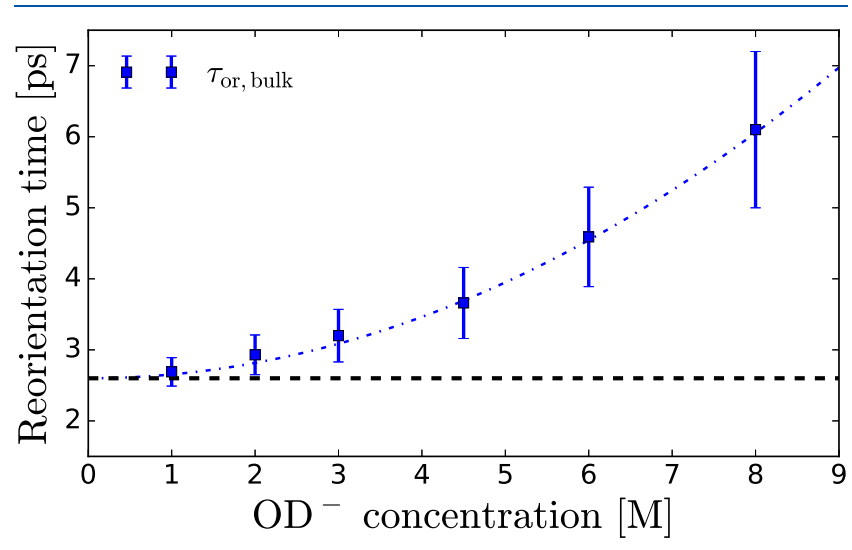

Figure 7. Reorientation time constant of bulk-like HDO molecules in $\mathrm{NaOD} / \mathrm{D}_{2} \mathrm{O}$ solutions as a function of the $\mathrm{OD}^{-}$concentration (blue squares). Bulk-like HDO molecules imply HDO molecules that are not directly hydrogen bonded to $\mathrm{OD}^{-}$ions, that is, HDO molecules outside the first hydration shell of the $\mathrm{OD}^{-}$ions. The dashed black curve shows the molecular reorientation time constant of bulk neat water. The dashed blue curve is a guide to the eye.

from $2.7 \pm 0.2$ ps to $6 \pm 1 \mathrm{ps}$, in the concentration range of $1-$ $8 \mathrm{M}$, indicating an effective slowing down of HDO molecules beyond the immediate solvation shell of hydroxide ions. It should be noted that in the modeling, it was assumed that the bulk-like $\mathrm{OH}$ groups show uniform reorientation dynamics, which is not necessarily correct. In fact, there may be a distribution of reorientation time constants. The values reported in Figure 7 should thus be considered as the average reorientation time constants of all the water molecules outside the cores of the hydroxide hydration structures.

\section{DISCUSSION}

It is interesting to compare the present findings with the results of previous studies of the effect of hydroxide ions on the dynamics of water using other techniques. The molecular reorientation of water aqueous hydroxide solutions has also been studied with dielectric relaxation spectroscopy, both in the $\mathrm{GHz}$ domain ${ }^{29}$ and in the $\mathrm{THz}$ domain with time-domain $\mathrm{THz}$ spectroscopy. ${ }^{32}$ Dielectric relaxation studies of hydroxide solutions in the $\mathrm{GHz}$ domain are complicated by the large ionic conductivity of the ions, which obscures the dielectric relaxation modes that represent the reorientation of the water molecules. As a result, dielectric relaxation studies in the $\mathrm{GHz}$ domain have only been performed for hydroxide solutions with concentrations up to $2 \mathrm{M}^{29}$ In this study, no frequency shift of the main relaxation mode of water was observed, meaning that the average reorientation time constant does not significantly change, in agreement with our results for concentrations up to $2 \mathrm{M}$. In dielectric relaxation studies using time-domain $\mathrm{THz}$ spectroscopy, the ionic conductivity forms a less important contribution, and thus, hydroxide solutions with concentrations up to $5 \mathrm{M}$ could be studied with this technique. ${ }^{32}$ However, with time-domain $\mathrm{THz}$ spectroscopy, only the highfrequency wing of the main relaxation mode can be observed, and no information on the potential change of the reorientation time constant of the water molecules could be obtained.

The molecular reorientation of water in hydroxide solutions has also been studied with nuclear magnetic resonance (NMR) spectroscopy. ${ }^{28}$ In this study, the longitudinal spin relaxation time of deuterons in hydroxide solutions was measured over a wide concentration range and at different temperatures. In the limit of motional narrowing, the longitudinal spin relaxation is proportional to the reorientation time of the water molecules. The longitudinal spin relaxation time was observed to show a similar nonlinear increase with an increasing hydroxide concentration as we observe for the reorientation time of the $\mathrm{OH}$ groups of HDO molecules. The results of the two studies cannot be quantitatively compared, as the longitudinal spin relaxation time is an average over all water molecule in the solution, including water molecules in the first hydration shell of the $\mathrm{OD}^{-}$ions, whereas the time constants shown in Figure 7 exclusively represent the reorientation time constants of water molecules outside the first hydration shell.

It is also interesting to compare the characteristic length scale of the solute-solvent interaction that is probed in the present study with the length scales that are probed with other spectroscopic techniques, such as Raman multivariate curve resolution (Raman MCR), second-harmonic scattering (SHS), dielectric relaxation spectroscopy, and NMR. In Raman MCR, the vibrational frequencies of solvent molecules in the first hydration shell of a solute are distinguished from the response of the other solvent molecules. ${ }^{57}$ These vibrational frequencies are largely dependent on the local (hydrogen-bond) interactions and thus constitute a highly local probe of the solute-solvent interactions. The length scale that is probed with Raman MCR is similar to the length scale that is probed by measuring the vibrational energy relaxation of the $\mathrm{OH}$ stretch vibrations with ultrafast mid-IR spectroscopy, as the rate of this relaxation is also largely determined by the hydrogen bonds that are donated and accepted by the probed $\mathrm{OH}$ group.

SHS of aqueous solutions is a relatively new technique to probe the orientational correlation of water molecules. ${ }^{58-60}$ The orientational correlation and thus the SHS signal change upon the addition of ions. The length scale that is probed with SHS is still a point of debate but, as the signal relies on the orientational correlation of the hydrogen-bond network, is likely much larger than that of vibrational energy relaxation 
and Raman MCR. Dielectric relaxation spectroscopy ${ }^{29,32}$ and NMR spectroscopy ${ }^{28}$ are mostly sensitive to average or collective reorganization processes of the hydrogen-bond network. Dielectric relaxation spectroscopy and NMR spectroscopy thus probe the solution over a similar length scale as the measurement of the anisotropy of excited $\mathrm{OH}$ stretch vibrations with polarization-resolved ultrafast mid-IR spectroscopy. This length scale is much larger than that of vibrational energy relaxation and Raman MCR.

Figure 7 shows that the reorientation time constant exhibits a superlinear dependence on the hydroxide concentration. This behavior differs from that of water in ordinary salt solutions, for which it has been observed that the slowing down effect of anions such as $\mathrm{Cl}^{-}, \mathrm{Br}^{-}$, and $\mathrm{I}^{-}$on the reorientation of water hydroxyl groups tends to saturate at higher salt concentrations. ${ }^{48,61,62}$ This saturation has been explained from the fact that at high concentrations, the hydration shells start to overlap. This latter conclusion also implies that the slowing down effect is dominated by the hydrogen bonds that are donated by water hydroxyl groups to the anion and that the slowing down effect is hardly influenced by other nearby ions. An exception is solutions of salts where both the cation and the anion are strongly hydrated like $\mathrm{MgSO}_{4}{ }^{61}$ In this case, solventseparated ion pairs are created with a strong electric field that locks the dynamics of the hydrogen-bonded water in between the ions, thus leading to a much stronger slowing down effect than for the cases where strongly hydrating ions are combined with weakly hydrating counter ions, as in $\mathrm{Mg}\left(\mathrm{ClO}_{4}\right)_{2}$ or $\mathrm{CsSO}_{4}$.

It would be interesting to compare the effect of hydroxide ions on the reorientation dynamics of water molecules with the effect of protons on these dynamics. Unfortunately, this latter effect has only been studied up to a proton concentration of 2 M. ${ }^{47}$ A complication in studying the effect of high concentrations of protons is that the negatively charged counter ions usually have a strong slowing down effect on the water reorientation dynamics. ${ }^{48,61,62} \mathrm{Up}$ to a proton concentration of $2 \mathrm{M}$, no measurable effect was observed on the reorientation dynamics of the water molecules that were not directly involved in the proton hydration structure. ${ }^{47}$ However, it cannot be excluded that at higher proton concentrations, a similar effect on the water reorientation dynamics may arise as observed for hydroxide.

The superlinear dependence of the reorientation time constant on the concentration indicates that the hydroxide ions have a cooperative effect on the reorientation dynamics of water molecules. This cooperativity can be explained from the fact that each hydroxide ion induces a local strengthening of the hydrogen bonds and an enhancement of the polarization of a number of surrounding water molecules. The thus affected water molecules will interact more strongly with other nearby hydroxide ions, leading to a mutual enhancement of the strengthening of the hydrogen bonds and a cooperative stiffening of the hydrogen-bond network.

A superlinear dependence of the reorientation time constant of water molecules on the sugar concentration was also observed in a recent study of the reorientation of water molecules in aqueous sugar solutions. ${ }^{63,64}$ The hydroxyl groups of the sugar molecules donate hydrogen bonds to the surrounding water molecules, and it was found that the slowing down of the water molecules is strongly enhanced in case they belong to two or more sugar hydration shells.

\section{CONCLUSIONS}

We have investigated the reorientation dynamics of HDO molecules in $\mathrm{NaOD} / \mathrm{D}_{2} \mathrm{O}$ solutions by probing the anisotropy of the transient absorption change resulting from the excitation of the $\mathrm{OH}$ stretch vibrations with polarization-resolved femtosecond-IR spectroscopy. Thanks to the distinctive redshifted and fast relaxation of $\mathrm{OH}$ stretch vibrations in the core of the $\mathrm{OD}^{-}$hydration structures, we can spectrally distinguish the dynamics of the $\mathrm{OH}$ stretch vibrations outside this core from the dynamics of the core $\mathrm{OH}$ stretch vibrations.

This spectral distinction enables us to selectively determine the reorientation dynamics of water molecules outside the core of the $\mathrm{OD}^{-}$hydration structures. We find that the reorientation time of these bulk-like hydroxyl groups increases with the concentration of $\mathrm{OD}^{-}$ions, from $2.7 \pm 0.2$ to $6 \pm 1$ ps, in the concentration range of $1-8 \mathrm{M}$, indicating an effective slowing down of $\mathrm{HDO}$ molecules beyond the immediate solvation shell of hydroxide ions. This increase is superlinear, which implies that hydroxide ions have little effect on the hydrogen-bond network at low concentrations, that is, when the $\mathrm{OD}^{-}$ions are sufficiently separated. At a high $\mathrm{OD}^{-}$concentration, the reorientation dynamics of the water molecules are cooperatively affected by multiple $\mathrm{OD}^{-}$ions, turning the solution in a semi-rigid hydrogen-bond network.

\section{ASSOCIATED CONTENT}

\section{Supporting Information}

The Supporting Information is available free of charge at https://pubs.acs.org/doi/10.1021/acs.jpcb.0c03614.

Description of the mathematical approach for the spectral decomposition; vibrational relaxation mechanism and model to describe the molecular reorientation dynamics; table of the numerical values including optimal fitting parameters and covariance matrix; and plots showing the comparison of the mathematical model with the experimental data at different ion concentrations and different delay times (PDF)

\section{AUTHOR INFORMATION}

\section{Corresponding Authors}

Sander Woutersen - Van 't Hoff Institute for Molecular Sciences, University of Amsterdam, 1098 XH Amsterdam, Netherlands; 이이.org/0000-0003-4661-7738; Email: s.woutersen@uva.nl

Huib J. Bakker - AMOLF, 1098 XG Amsterdam, Netherlands; ○ orcid.org/0000-0003-1564-5314; Email: h.bakker@ amolf.nl

\section{Authors}

Roberto Cota - Van 't Hoff Institute for Molecular Sciences, University of Amsterdam, 1098 XH Amsterdam, Netherlands; AMOLF, 1098 XG Amsterdam, Netherlands; (1) orcid.org/ 0000-0002-5270-6158

Eliane P. van Dam - AMOLF, 1098 XG Amsterdam, Netherlands; 이이.org/0000-0002-3051-8922

Complete contact information is available at:

https://pubs.acs.org/10.1021/acs.jpcb.0c03614

\section{Notes}

The authors declare no competing financial interest. 


\section{ACKNOWLEDGMENTS}

The authors kindly thank Hinco Schoenmakers and Hans Sanders for technical support and Alexander Korotkevich and Aleksandr Sofronov for fruitful discussions. This work is part of the research program of the Netherlands Organization for Scientific Research (NWO), grant number 12PR2989.

\section{REFERENCES}

(1) Kresge, A. J. What Makes Proton Transfer Fast? Acc. Chem. Res. 1975, 8, 354-360.

(2) Cukierman, S.; Quigley, E. P.; Crumrine, D. S. Proton conduction in gramicidin $\mathrm{A}$ and in its dioxolane-linked dimer in different lipid bilayers. Biophys. J. 1997, 73, 2489-2502.

(3) Pomès, R. Theoretical studies of the Grotthuss mechanism in biological proton wires. Isr. J. Chem. 1999, 39, 387-395.

(4) Brewer, M. L.; Schmitt, U. W.; Voth, G. A. The Formation and Dynamics of Proton Wires in Channel Environments. Biophys. J. 2001, 80, 1691-1702.

(5) Pomès, R.; Roux, B. Molecular Mechanism of $\mathrm{H}^{+}$Conduction in the Single-File Water Chain of the Gramicidin Channel. Biophys. J. 2002, 82, 2304-2316.

(6) Cukierman, S.; Quigley, E. P.; Crumrine, D. S. Proton conduction in gramicidin $\mathrm{A}$ and in its dioxolane-linked dimer in different lipid bilayers. Biophys. J. 1997, 73, 2489-2502.

(7) Swanson, J. M. J.; Maupin, C. M.; Chen, H.; Petersen, M. K.; Xu, J.; Wu, Y.; Voth, G. A. Proton solvation and transport in aqueous and biomolecular systems: Insights from computer simulations. J. Phys. Chem. B 2007, 111, 4300-4314.

(8) Ball, P. Water-an enduring mystery. Nature 2008, 452, 291292.

(9) Hibbs, M. R.; Hickner, M. A.; Alam, T. M.; McIntyre, S. K.; Fujimoto, C. H.; Cornelius, C. J. Transport Properties of Hydroxide and Proton Conducting Membranes. Chem. Mater. 2008, 20, 25662573.

(10) Pan, J.; Chen, C.; Zhuang, L.; Lu, J. Designing Advanced Alkaline Polymer Electrolytes for Fuel Cell Applications. Acc. Chem. Res. 2012, 45, 473-481.

(11) Deng, H.; Huo, S.; Chang, Y.; Zhou, Y.; Jiao, K. Transient analysis of alkaline anion exchange membrane fuel cell anode. Int. J. Hydrogen Energy 2013, 38, 6509-6525.

(12) Ensing, W.; Hunger, J.; Ottosson, N.; Bakker, H. J. On the orientational mobility of water molecules in proton and sodium terminated nafion membranes. J. Phys. Chem. C 2013, 117, 1293012935.

(13) Mani, D.; De Tudela, R. P.; Schwan, R.; Pal, N.; Körning, S.; Forbert, H.; Redlich, B.; Van Der Meer, A. F. G.; Schwaab, G.; Marx, D.; Havenith, M. Acid solvation versus dissociation at "stardust conditions": Reaction sequence matters. Sci. Adv. 2019, 5, No. eaav8179.

(14) Agmon, N. The Grotthuss mechanism. Chem. Phys. Lett. 1995, 244, 456-462.

(15) Marx, D.; Tuckerman, M. E.; Hutter, J.; Parrinello, M. The nature of the hydrated excess proton in water. Nature 1999, 397, 601-604.

(16) Mohammed, O. F.; Pines, D.; Dreyer, J.; Pines, E.; Nibbering, E. T. J. Sequential Proton Transfer Through Water Bridges in AcidBase Reactions. Science 2005, 310, 83-86.

(17) Woutersen, S.; Bakker, H. J. Ultrafast vibrational and structural dynamics of the proton in liquid water. Phys. Rev. Lett. 2006, 96, 138305.

(18) Markovitch, O.; Agmon, N. Structure and Energetics of the Hydronium Hydration Shells. J. Phys. Chem. A 2007, 111, 22532256.

(19) Cox, M. J.; Bakker, H. J. Parallel proton transfer pathways in aqueous acid-base reactions. J. Chem. Phys. 2008, 128, 174501.

(20) Berkelbach, T. C.; Lee, H.-S.; Tuckerman, M. E. Concerted Hydrogen-Bond Dynamics in the Transport Mechanism of the
Hydrated Proton: A First-Principles Molecular Dynamics Study. Phys. Rev. Lett. 2009, 103, 238302.

(21) Maupin, C. M.; Aradi, B.; Voth, G. A. The Self-Consistent Charge Density Functional Tight Binding Method Applied to Liquid Water and the Hydrated Excess Proton: Benchmark Simulations. J. Phys. Chem. B 2010, 114, 6922-6931.

(22) Lee, S. H.; Rasaiah, J. C. Proton transfer and the mobilities of the $\mathrm{H}^{+}$and $\mathrm{OH}^{-}$ions from studies of a dissociating model for water. $J$. Chem. Phys. 2011, 135, 124505.

(23) Meng, X.; Guo, J.; Peng, J.; Chen, J.; Wang, Z.; Shi, J.-R.; Li, X.Z.; Wang, E.-G.; Jiang, Y. Direct visualization of concerted proton tunnelling in a water nanocluster. Nat. Phys. 2015, 11, 235-239.

(24) Wolke, C. T.; Fournier, J. A.; Dzugan, L. C.; Fagiani, M. R.; Odbadrakh, T. T.; Knorke, H.; Jordan, K. D.; McCoy, A. B.; Asmis, K. R.; Johnson, M. A. Spectroscopic snapshots of the proton-transfer mechanism in water. Science 2016, 354, 1131-1135.

(25) Biswas, R.; Carpenter, W.; Fournier, J. A.; Voth, G. A.; Tokmakoff, A. IR spectral assignments for the hydrated excess proton in liquid water. J. Chem. Phys. 2017, 146, 154507.

(26) Daly, C. A.; Streacker, L. M.; Sun, Y.; Pattenaude, S. R.; Hassanali, A. A.; Petersen, P. B.; Corcelli, S. A.; Ben-Amotz, D. Decomposition of the Experimental Raman and Infrared Spectra of Acidic Water into Proton, Special Pair, and Counterion Contributions. J. Phys. Chem. Lett. 2017, 8, 5246-5252.

(27) Chen, M.; Zheng, L.; Santra, B.; Ko, H.-Y.; DiStasio Jr, R. A.; Klein, M. L.; Car, R.; Wu, X. Hydroxide diffuses slower than hydronium in water because its solvated structure inhibits correlated proton transfer. Nat. Chem. 2018, 10, 413-419.

(28) Chizhik, V. I.; Mikhailov, V. I.; Su, P. C. NMR relaxation data on the microstructure of aqueous solutions of alkali-metal salts and hydroxides. Theor. Exp. Chem. 1987, 22, 480-483.

(29) Buchner, R.; Hefter, G.; May, P. M.; Sipos, P. Dielectric relaxation of dilute aqueous $\mathrm{NaOH}, \mathrm{NaAl}(\mathrm{OH})_{4}$, and $\mathrm{NaB}(\mathrm{OH})_{4}$. J. Phys. Chem. B 1999, 103, 11186-11190.

(30) Nienhuys, H.-K.; Lock, A. J.; van Santen, R. A.; Bakker, H. J. Dynamics of water molecules in an alkaline environment. J. Chem. Phys. 2002, 117, 8021-8029.

(31) Roberts, S. T.; Petersen, P. B.; Ramasesha, K.; Tokmakoff, A.; Ufimtsev, I. S.; Martinez, T. J. Observation of a Zundel-like transition state during proton transfer in aqueous hydroxide solutions. Proc. Natl. Acad. Sci. U. S. A. 2009, 106, 15154-15159.

(32) Hunger, J.; Liu, L.; Tielrooij, K.-J.; Bonn, M.; Bakker, H. Vibrational and orientational dynamics of water in aqueous hydroxide solutions. J. Chem. Phys. 2011, 135, 124517.

(33) Mandal, A.; Ramasesha, K.; De Marco, L.; Tokmakoff, A. Collective vibrations of water-solvated hydroxide ions investigated with broadband 2DIR spectroscopy. J. Chem. Phys. 2014, 140, 204508.

(34) Mandal, A.; Tokmakoff, A. Vibrational dynamics of aqueous hydroxide solutions probed using broadband 2DIR spectroscopy. J. Chem. Phys. 2015, 143, 194501.

(35) Agmon, N. Mechanism of hydroxide mobility. Chem. Phys. Lett. 2000, 319, 247-252.

(36) Asthagiri, D.; Pratt, L. R.; Kress, J. D.; Gomez, M. A. The hydration state of $\mathrm{HO}^{-}$(aq). Chem. Phys. Lett. 2003, 380, 530-535.

(37) Tuckerman, M. E.; Marx, D.; Parrinello, M. The nature and transport mechanism of hydrated hydroxide ions in aqueous solution. Nature 2002, 417, 925-929.

(38) Chen, B.; Ivanov, I.; Park, J. M.; Parrinello, M.; Klein, M. L. Solvation Structure and Mobility Mechanism of $\mathrm{OH}^{-}$: A CarParrinello Molecular Dynamics Investigation of Alkaline Solutions. J. Phys. Chem. B 2002, 106, 12006-12016.

(39) Sun, X.; Yoo, S.; Xantheas, S. S.; Dang, L. X. The reorientation mechanism of hydroxide ions in water: A molecular dynamics study. Chem. Phys. Lett. 2009, 481, 9-16.

(40) Bucher, D.; Gray-Weale, A.; Kuyucak, S. Ab Initio Study of Water Polarization in the Hydration Shell of Aqueous Hydroxide: Comparison between Polarizable and Nonpolarizable Water Models. J. Chem. Theory Comput. 2010, 6, 2888-2895. 
(41) Hassanali, A.; Prakash, M. K.; Eshet, H.; Parrinello, M. On the recombination of hydronium and hydroxide ions in water. Proc. Natl. Acad. Sci. U. S. A. 2011, 108, 20410-20415.

(42) Roberts, S. T.; Mandal, A.; Tokmakoff, A. Local and Collective Reaction Coordinates in the Transport of the Aqueous Hydroxide Ion. J. Phys. Chem. B 2014, 118, 8062-8069.

(43) Roberts, S. T.; Ramasesha, K.; Petersen, P. B.; Mandal, A.; Tokmakoff, A. Proton transfer in concentrated aqueous hydroxide visualized using ultrafast infrared spectroscopy. J. Phys. Chem. A 2011, 115, 3957-3972.

(44) Thøgersen, J.; Jensen, S. K.; Petersen, C.; Keiding, S. R. Reorientation of hydroxide ions in water. Chem. Phys. Lett. 2008, 466, $1-5$.

(45) Kropman, M. F.; Bakker, H. J. Dynamics of water molecules in aqueous solvation shells. Science 2001, 291, 2118-2120.

(46) Kropman, M. F.; Nienhuys, H.-K.; Bakker, H. J. Real-Time Measurement of the Orientational Dynamics of Aqueous Solvation Shells in Bulk Liquid Water. Phys. Rev. Lett. 2002, 88, 077601.

(47) Tielrooij, K. J.; Timmer, R. L. A.; Bakker, H. J.; Bonn, M. Structure dynamics of the proton in liquid water probed with terahertz time-domain spectroscopy. Phys. Rev. Lett. 2009, 102, 198303.

(48) van der Post, S. T.; Scheidelaar, S.; Bakker, H. J. Femtosecond study of the effects of ions on the reorientation dynamics of water. $J$. Mol. Liq. 2012, 176, 22-28.

(49) van der Post, S. T.; Bakker, H. J. The combined effect of cations and anions on the dynamics of water. Phys. Chem. Chem. Phys. 2012, $14,6280-6288$

(50) Rezus, Y. L. A.; Bakker, H. J. On the orientational relaxation of HDO in liquid water. J. Chem. Phys. 2005, 123, 114502.

(51) Liu, L.; Hunger, J.; Bakker, H. J. Energy Relaxation Dynamics of the Hydration Complex of Hydroxide. J. Phys. Chem. A 2011, 115, 14593-14598.

(52) Timmer, R. L. A.; Tielrooij, K. J.; Bakker, H. J. Vibrational Förster transfer to hydrated protons. J. Chem. Phys. 2010, 132, 194504.

(53) Rezus, Y. L. A.; Bakker, H. J. Orientational dynamics of isotopically diluted $\mathrm{H}_{2} \mathrm{O}$ and $\mathrm{D}_{2} \mathrm{O}$. J. Chem. Phys. 2006, 125, 144512.

(54) Roberts, S. T.; Loparo, J. J.; Tokmakoff, A. Characterization of spectral diffusion from two-dimensional line shapes. J. Chem. Phys. 2006, 125, 084502.

(55) Steinel, T.; Asbury, J. B.; Zheng, J.; Fayer, M. D. Watching hydrogen bonds break: A transient absorption study of water. J. Phys. Chem. A 2004, 108, 10957-10964.

(56) Park, S.; Odelius, M.; Gaffney, K. J. Ultrafast Dynamics of Hydrogen Bond Exchange in Aqueous Ionic Solutions. J. Phys. Chem. B 2009, 113, 7825-7835.

(57) Fega, K. R.; Wilcox, A. S.; Ben-Amotz, D. Application of Raman multivariate curve resolution to solvation-shell spectroscopy. Appl. Spectrosc. 2012, 66, 282-288.

(58) Shelton, D. P. Long-range orientation correlation in water. J. Chem. Phys. 2014, 141, 224506.

(59) Chen, Y.; Okur, H. I.; Gomopoulos, N.; Macias-Romero, C.; Cremer, P. S.; Petersen, P. B.; Tocci, G.; Wilkins, D. M.; Liang, C.; Ceriotti, M.; Roke, S. Electrolytes induce long-range orientational order and free energy changes in the $\mathrm{H}$-bond network of bulk water. Sci. Adv. 2016, 2, No. e1501891.

(60) Duboisset, J.; Brevet, P.-F. Salt-induced Long-to-Short Range Orientational Transition in Water. Phys. Rev. Lett. 2018, 120, 263001.

(61) Tielrooij, K. J.; Garcia-Araez, N.; Bonn, M.; Bakker, H. J. Cooperativity in ion hydration. Science 2010, 328, 1006-1009.

(62) van der Post, S. T.; Tielrooij, K.-J.; Hunger, J.; Backus, E. H. G.; Bakker, H. J. Femtosecond study of the effects of ions and hydrophobes on the dynamics of water. Faraday Discuss. 2013, 160, $171-189$.

(63) Groot, C. C. M.; Bakker, H. J. Hydration dynamics of aqueous glucose probed with polarization-resolved fs-IR spectroscopy. J. Chem. Phys. 2014, 140, 234503.
(64) Groot, C. C. M.; Bakker, H. J. A femtosecond mid-infrared study of the dynamics of water in aqueous sugar solutions. Phys. Chem. Chem. Phys. 2015, 17, 8449-8458. 